\title{
GUEST EDITORIAL special issue on real-time perceptual- inspired imaging systems with computational science and aesthetics
}

\author{
Sanghyun Seo ${ }^{1} \cdot$ Bo-Wei Chen $^{2} \cdot$ Periklis Chatzimisios $^{3} \cdot{\text { Seungmin } \text { Rho }^{1}}^{1}$
}

Published online: 21 September 2017

(C) Springer-Verlag GmbH Germany 2017

\section{Introduction}

The objective of a perceptual-inspired imaging system is to explore consumer-focused hardware and software for applications such as recognition, tracking, visualization, and augmented reality. Perceptual approaches have been widely used in many areas of multimedia and visual information processing. As a multimedia system produces sounds, images, and videos that are ultimately perceived by a human, it is essential to account for how the human visual system (HVS) processes this information. The HVS is complex, exhibiting many nonlinearities as well as feedback and is only partially understood. While this poses a challenge, it can also be seen as an opportunity that can be exploited in multimedia systems with computational science and aesthetics. These systems bridge the analytic and synthetic by integrating aspects of computer science, psychology, emotional engineering, and cognitive science. It can also include fine, applied, and media arts.

Sanghyun Seo

shseo75@gmail.com

Bo-Wei Chen

dennisbwc@gmail.com

Periklis Chatzimisios

pchatzimisios@ieee.org

Seungmin Rho

smrho@sungkyul.edu

1 Division of Media Software, Sungkyul University, Anyang, Republic of Korea

2 Princeton University, Princeton, NJ, USA

3 Department of Informatics, Alexander Technological Educational Institute of Thessaloniki (ATEITHE), Sindos, Greece
The goal of this special issue is to explore real-time models, algorithms, and technologies needed to enable perceptual-inspired multimedia systems. This is intended to foster the dissemination of state-of-the-art research related to "Real-Time Perceptual-inspired Imaging Systems with Computational Science and Aesthetics" that can invoke human emotional responses.

In the following section, we present a brief overview of each manuscript and then conclude this guest editorial.

\section{Real-time perceptual-inspired imaging systems}

The first paper, entitled "Real-time 3D fluid simulation digital art using BCI," by Cho and Hong [4] proposed a system for digital art that simulated real-time 3D fluid using a brain-computer interface. The system interacts with user's emotion, therefore, immersing the user in their digital art work. To determine a user's emotional state, they measured the user's electroencephalography signal using MindSet. In their real-time fluid simulation system implemented on Unity3D, the properties of fluids, such as scale, speed, and appearance, varied according to the user's emotional state.

The second paper, entitled "Saliency weighted graphs for efficient visual content description and their applications in real-time image retrieval systems," by Ahmad et al. [1] proposed a graph-based image representation model with an efficient matching algorithm for real-time image retrieval. To achieve a representation closer to the human perception model, they extracted salient color and edge features and utilized them to generate a salient featured weighted graph. With a lightweight graph-matching procedure, this graph was used to retrieve a relevant image. 
Thus, their approach achieved both effective and efficient image retrieval performance.

The third paper, entitled "Accurate and efficient shape matching approach using vocabularies of multi-feature space representations," by Khalid et al. [10] proposed an approach for efficient shape retrieval using a combination of Fourier descriptors and inner-distance-based shape context features. They preprocessed the shapes to account for the variation in scale and the presence of cracks, and generated multiple feature space representations of shapes using a hierarchical indexing structure to retrieve shapes both efficiently and accurately. To enable real-time imagebased shape analysis, they also presented a quantization approach for generating a vocabulary of feature space representation of shapes.

The fourth paper, entitled "Real-time video watermarking techniques robust against re-encoding," by Yoo and Kim [17] addressed implementation issues for realtime watermark embedding schemes. They presented a real-time watermarking codec that is robust against re-encoding attacks for high-definition videos. In their study, they provided performance optimization guidelines and a simplified human visual system-based watermarking method for fast and robust watermark embedding.

The fifth paper, entitled "A real-time image dehazing method considering dark channel and statistics features," by Yang et al. [16] proposed a novel real-time image dehazing algorithm based on both histogram optimization and dark channel prior. In their algorithm, they used histogram optimization to stretch the contrast of the image and used the dark channel prior to estimate the haze intensity. To achieve real-time performance, they proposed an approach that decomposed the image into high-frequency components and low-frequency components by utilizing a wavelet transformation, and performed dehazing only on the low-frequency components.

The sixth paper, entitled "Real-time imaging-based assessment model for improving teaching performance and student experience in e-learning," by Farhan et al. [6] explored student-focused software applications that track and recognize students and teachers in the e-Learning environment by presenting a real-time imaging-based assessment model. To track a student's visual attention and a teacher's visual engagement, they proposed algorithms for real-time scoring visual attention and engagement.

The seventh paper, entitled "Real-time recognition of cattle using animal biometrics," by Kumar et al. [13] proposed a novel muzzle point recognition system for identifying individual cattle in the real time. The proposed system captures images of cattle automatically through surveillance cameras, obtains muzzle features from segmented muzzle point images, and uses the features to match muzzle points using One-Shot Similarity, distance matrices-based matching, and one-class support vector machine classification. The used approach yields a $96.87 \%$ recognition accuracy rate with real-time performance.

The eighth paper, entitled "Automated wireless video surveillance: an evaluation framework," by Alsmirat et al. [2] proposed a realistic wireless automated video surveillance system (AVS) simulation framework. The proposed framework built by extending NS-3, which is an open-source network simulator, provides a realistic MJPEG application layer that streams real video over a simulation network. It also supports the evaluation of cross-layer solutions that manage many factors over different layers of AVS systems in wireless network.

The ninth paper, entitled "QoS optimal real-time video streaming in distributed wireless image-sensing platforms," by Kim and Ryu [11] proposed a real-time video streaming method for distributed wireless image-sensing platforms that used a multi-hop routing protocol assisted by multi-antenna relays. The proposed method optimizes the combined QoS of the individual wireless transmissions of different video streams subject to the constraints of each stream. They also considered QoS awareness with a millimeter wave to support real-time wireless video streaming applications.

The tenth paper, entitled "Real-time, large-scale duplicate image detection method based on multi-feature fusion," by Chen et al. [3] proposed a real-time large-scale duplicate image detection method using multi-feature fusion for improving retrieval accuracy. To generate image signatures, the proposed method uses a perception hash, reducing the search time of images. It also uses blockaverage grayscale features and Haar wavelet features to conduct multiple filtering. Consequently, the method achieves improved retrieval accuracy.

The eleventh paper, entitled "Binocular mobile augmented reality based on stereo camera tracking," by Park et al. [14] presented an augmented reality framework for mobile stereo display. By modifying edge-based 3D object tracking, they obtained a joint camera pose estimation in stereo camera tracking that allows the precise registration of the real and the virtual worlds and consistent augmentation across both views. Despite heavy computations for stereo camera tracking and image warping, interactive performance is achieved in the framework by utilizing GPU and multi-threading.

The twelfth paper, entitled "Perceptually inspired realtime artistic style transfer for video stream," by Kang et al. [9] presented a real-time texture transfer method for artistic stylization of video stream. To achieve real-time performance, they proposed a parallel framework using a T-shaped kernel and computed downscaled motion between frames. Moreover, to enhance the artistic effect, they utilized the visual saliency as the level of abstraction. 
The thirteenth paper, entitled "Real-time tonal depiction method by reaction-diffusion mask," by Jho and Lee [7] presented a technique for real-time tonal depiction with the reaction-diffusion (R-D) model, which is usually used to depict skin patterns of animals. To improve the large time consumption of the R-D model, they proposed a new mask, which enables real-time processing when applied independently to each pixel. The proposed method generates almost the same result as the original model while achieving dramatic improvement in the computation speed.

The fourteenth paper, entitled "Spatial and temporal pyramid-based real-time gesture recognition," by Jiang et al. [8] presented a real-time hand gesture recognition (HGR) system using Kinect. They proposed spatial and temporal pyramid-based real-time gesture recognition that takes into account both the simultaneous and sequential organization of gestures. To improve the effectiveness and accuracy of HGR, a spatial pyramid is applied to segment gesture sequences into linguistic units, and a temporal pyramid is used to obtain a time-related histogram for each gesture. Utilizing the two proposed pyramids, they obtained more comprehensive information of human gestures in real time.

The fifteenth paper, entitled "DBN wavelet transform denoising method in soybean straw composition based on near-infrared rapid detection," by Kong et al. [12] presented a method for rapidly detecting a soybean straw's internal components using near-infrared spectroscopy technology. They combined NIR spectroscopy with chemometric methods to establish the partial least square prediction model.

The sixteenth paper, entitled "Game theoretic approach for real-time data dissemination and offloading in vehicular ad hoc networks," by Dua et al. [5] proposed a game theoretic approach that provides a global solution for mobile data offloading by calculating the utility of nearby vehicles and APs based on real-time parameters. They thereby achieved higher message progress and dissemination speed in the proposed scheme.

The last paper, entitled "Uncertain clustering algorithms based on rough and fuzzy sets for real-time image segmentation," by Shi et al. [15] aimed to represent and manage uncertainties in the field of pattern recognition, especially in clustering. To achieve this, they proposed an adaptive selection strategy for the weighted parameter in rough set-based clustering algorithms.

\section{Conclusion}

Finally, we would like to thank the Chief Editors of the Journal of Real-Time Image Processing, Nasser Kehtarnavaz and Matthias F. Carlsohn, and the entire editorial staff for their valuable support throughout the preparation and publication of this special issue. We would like to thank all the authors for their contributions to this special issue. We would also like to thank the external reviewers for their immense help in reviewing the manuscripts. We hope that the selected papers will contribute to the ongoing research in convergence of real-time imaging in academia, industry, and other interested agencies and organizations.

\section{References}

1. Ahmad, J., Sajjad, M., Mehmood, I., Rho, S., Baik, S.W.: Saliency-weighted graphs for efficient visual content description and their applications in real-time image retrieval systems. J. Real Time Image Process. (2015). doi:10.1007/s11554-015-0536-0

2. Alsmirat, M.A., Jararweh, Y., Obaidat, I., Gupta, B.B.: Automated wireless video surveillance: an evaluation framework. J. Real Time Image Process. (2016). doi:10.1007/s11554-0160631-x

3. Chen, M., Li, Y., Zhang, Z., Hsu, C.H., Wang, S.: Real-time, large-scale duplicate image detection method based on multifeature fusion. J. Real Time Image Process. (2016). doi:10.1007/ s11554-016-0632-9

4. Cho, O.H., Hong, S.: Real-time $3 \mathrm{~d}$ fluid simulation digital art using BCI. J. Real Time Image Process. (2015). doi:10.1007/ s11554-015-0546-y

5. Dua, A., Kumar, N., Bawa, S.: Game theoretic approach for realtime data dissemination and offloading in vehicular ad hoc networks. J. Real Time Image Process. (2016). doi:10.1007/s11554016-0615-x

6. Farhan, M., Aslam, M., Jabbar, S., Khalid, S., Kim, M.: Real-time imaging-based assessment model for improving teaching performance and student experience in e-learning. J. Real Time Image Process. (2017). doi:10.1007/s11554-016-0662-3

7. Jho, C.W., Lee, W.H.: Real-time tonal depiction method by reaction-diffusion mask. J. Real Time Image Process. (2016). doi:10.1007/s11554-016-0643-6

8. Jiang, F., Ren, J., Lee, C., Shi, W., Liu, S., Zhao, D.: Spatial and temporal pyramid-based real-time gesture recognition. J. Real Time Image Process. (2016). doi:10.1007/s11554-016-0620-0

9. Kang, D., Tian, F., Seo, S.: Perceptually inspired real-time artistic style transfer for video stream. J. Real Time Image Process. (2016). doi:10.1007/s11554-016-0612-0

10. Khalid, S., Sajjad, S., Jabbar, S., Chang, H.: Accurate and efficient shape matching approach using vocabularies of multi-feature space representations. J. Real Time Image Process. (2015). doi:10.1007/s11554-015-0545-z

11. Kim, J., Ryu, E.S.: QoS optimal real-time video streaming in distributed wireless image-sensing platforms. J. Real-Time Image Process. (2016). doi:10.1007/s11554-016-0629-4

12. Kong, Q., Cui, G., Yeo, S.S., Su, Z., Wang, J., Hu, F., Shen, W., Padigala, V.A.: DBN wavelet transform denoising method in soybean straw composition based on near-infrared rapid detection. J. Real Time Image Process. (2016). doi:10.1007/s11554016-0642-7

13. Kumar, S., Singh, S.K., Singh, R.S., Singh, A.K., Tiwari, S.: Real-time recognition of cattle using animal biometrics. J. Real Time Image Process. (2016). doi:10.1007/s11554-016-0645-4

14. Park, J., Seo, B.K., Park, J.I.: Binocular mobile augmented reality based on stereo camera tracking. J. Real Time Image Process. (2016). doi:10.1007/s11554-016-0640-9 
15. Shi, J., Lei, Y., Wu, J., Paul, A., Kim, M., Jeon, G.: Uncertain clustering algorithms based on rough and fuzzy sets for real-time image segmentation. J. Real Time Image Process. (2016). doi:10. 1007/s11554-016-0585-Z

16. Yang, J., Jiang, B., Lv, Z., Jiang, N.: A real-time image dehazing method considering dark channel and statistics features. J. Real Time Image Process. (2017). doi:10.1007/s11554-017-0671-x

17. Yoo, G., Kim, H.: Real-time video watermarking techniques robust against re-encoding. J. Real Time Image Process. (2015). doi:10.1007/s11554-015-0557-8

Sanghyun Seo received his B.S. degrees in Computer Science and Engineering from Chung-Ang University, Seoul, Korea, in 1998 and M.S. and Ph.D. degrees in GSAIM Dep at Chung-Ang University, Seoul, Korea, in 2000 and 2010. He was senior researcher at G-Inno System from 2002 to 2005 . He was the postdoctral researcher at Chung-Ang University, in 2010, and the postdoctral researcher at LIRIS Lab, Lyon 1 Unviersity from February 2011 to February 2013. He had worked at the ETRI (Electronics and Telecommunications Research Institute), DaeJeon, Korea, May 2013 to February 2016. Now, he is currently a faculty of Department of Media Software at Sungkyul University He has been a reviewer in Multimedia Tools and Applications (MTAP), Computer and Graphics UK (Elsevier), Journal of Supercomputing (JOS), Visual Computer (Springer), and Program Committee member in many international conferences and workshops and has edited a number of international journal special issues as a guest editor, such as Journal of Real-Time Image Processing, Journal of Internet Technology, and Multimedia Tools and Applications and so on. His research interests are in the area of computer graphics, nonphotorealistic rendering and animation, 3D GIS system, real-time rendering using GPU, AR (augmented reality), and game technology.

Bo-Wei Chen received the Ph.D. degree in electrical engineering from National Cheng Kung University (NCKU), Taiwan, in 2009. Until 2013, he was a Postdoctoral Research Fellow with the Department of Electrical Engineering, NCKU. He worked as a Postdoctoral Research Fellow with the Department of Electrical Engineering, Princeton University, Princeton, NJ, USA, during 2014-2015. He is currently a Senior Lecturer with the School of Information Technology, Monash University Sunway Campus. Dr. Chen serves as the Chair of the Signal Processing Chapter, IEEE Harbin Section. He was invited as a Guest Editor in ACM Transactions in Embedded Computing, Elsevier Journal of Neurocomputing, and Springer Multimedia Tools and Applications.

Periklis Chatzimisios serves as an Associate Professor and the Director of the Computing Systems, Security and Networks (CSSN)
Research Lab and Division Head in the Department of Informatics at the Alexander TEI of Thessaloniki (ATEITHE), Greece. He is currently a Visiting Fellow with the Faculty of Science and Technology, Bournemouth University (UK). In the past, he has been a Visiting Academic/Researcher with the University of Toronto (Canada) and the Massachusetts Institute of Technology (USA). He is the editor/author of eight books and more than 110 peer-reviewed papers and book chapters on the topics of performance evaluation and standardization activities of mobile/wireless communications, Internet of Things, and Big Data. His published research work has received more than 2200 citations by other researchers. Dr. Chatzimisios is/has been involved in several standardization and IEEE activities serving as a member of the Standards Development Board for the IEEE Communication Society (ComSoc), a member of the IEEE ComSoc Standards Program Development Board, a member of the IEEE ComSoC Education and Training Board, an Associate Editor for Global Online Communications Newsletter, Vice-Chair of the Emerging Technical Subcommittee on Big Data, and Secretary of the IEEE ComSoc Technical Committee in Information Infrastructure and Networking. Dr. Chatzimisios received the B.Sc. degree from ATEITHE (Greece) in 2000 and the Ph.D. degree from Bournemouth University (U.K.) in 2005.

Seungmin Rho received his Ph.D. degree in Computer Science from Ajou University, Korea in 2008. In 2008-2009, he was a Postdoctoral Research Fellow at the Computer Music Lab of the School of Computer Science in Carnegie Mellon University. Dr. Rho is currently a faculty of Department of Media Software at Sungkyul University. His current research interests include database, big data analysis, music retrieval, multimedia systems, machine learning, knowledge management, and computational intelligence. He has published more than 200 papers in refereed journals and conference proceedings in these areas. He has been involved in more than 20 conferences and workshops as various chairs and more than 30 conferences/workshops as a program committee member. He has been appointed as an Editor-in-Chief in Journal of Platform Technology since 2013. He has edited a number of international journal special issues as a guest editor, such as Enterprise Information Systems, Multimedia Systems, Information Fusion, ACM Transactions on Embedded Computing, Journal of Real-Time Image Processing, Future Generation Computer Systems, Engineering Applications of Artificial Intelligence, New Review of Hypermedia and Multimedia, Multimedia Tools and Applications, Personal and Ubiquitous Computing, Telecommunication Systems, Ad Hoc and Sensor Wireless Networks. 